\title{
New understanding of TFEB and autophagy in the development of endometriosis:review
}

\author{
qiuyu chen ${ }^{1}$, yu zhao ${ }^{1}$, sennan zhu ${ }^{1}$, yao chen ${ }^{1}$, Chenxi Dai ${ }^{1}$, Zongwen Liang ${ }^{1}$, and qiong \\ zhang $^{1}$
}

${ }^{1}$ Affiliation not available

October 26, 2020

\begin{abstract}
Endometriosis is a benign gynecologic disease, but it is similar in behavior to malignant tumors, so it is called "benign gynecologic cancer". At present, its pathogenesis is not fully understand. Autophagy has been the focus of recent research on various diseases, which has also been shown to have regulatory abnormalities in endometriosis. TFEB is a key regulator of autophagy. The upstream signal molecules and downstream effector proteins of TFEB have abnormal expression in endometriosis. So TFEB may be an effective target for controlling the development of endometriosis. This review aims to discuss the relationship between TFEB and endometriosis.
\end{abstract}

\section{Title}

New understanding of TFEB and autophagy in the development of endometriosis:review

running Title: TFEB and autophagy in endometriosis

\section{Authos}

Qiuyu Chen ${ }^{1,2^{*}}$, Sennan Zhu ${ }^{2 *}$, Yao Chen ${ }^{2}$, Chenxi Dai ${ }^{2}$, Zongwen Liang ${ }^{1}$, Qiong Zhang ${ }^{1,2 \#}$, Yu Zhao $^{1,2 \#}$. *Qiuyu Chen and Sennan Zhu contributed equally to this work and should be considered co-first Authors \#Qiong Zhang and Yu Zhao contributed equally to this work and should be considered co-corresponding authors

\section{Affiliations}

${ }^{1}$ Department of Obstetrics and Gynaecology, The Second Affiliated Hospital and Yuying Children's Hospital of Wenzhou Medical University, Wenzhou, Zhejiang 325000, China.

${ }^{2}$ The Second School of Medicine, Wenzhou Medical University, Zhejiang 325000, Wenzhou, China.

\section{Correspondent authors :}

$\mathrm{Yu}$ Zhao, Department of Obstetrics and Gynaecology, The Second Affiliated Hospital and Yuying Children's Hospital of Wenzhou Medical University, 306 Hualongqiao road, Wenzhou, 325000, China; Email:zhaoyu196035@163.com; Tel: 86-577- 88002230; Fax: 86-577-88832693.

Qiong Zhang, Department of Obstetrics and Gynaecology, The Second Affiliated Hospital and Yuying Children's Hospital of Wenzhou Medical University, 306 Hualongqiao road ,Wenzhou, 325000, China; Email:joan_zhang2002@sina.com; Tel: 86-577- 88002230; Fax: 86-577-88832693.

\section{Abstract:}


Endometriosis is a benign gynecologic disease, but it is similar in behavior to malignant tumors, so it is called "benign gynecologic cancer". At present, its pathogenesis is not fully understand. Autophagy has been the focus of recent research on various diseases, which has also been shown to have regulatory abnormalities in endometriosis. TFEB is a key regulator of autophagy. The upstream signal molecules and downstream effector proteins of TFEB have abnormal expression in endometriosis. So TFEB may be an effective target for controlling the development of endometriosis. This review aims to discuss the relationship between TFEB and endometriosis.

Keywords :autophagy, endometriosis, TFEB, ROS.

\section{Introduction :}

Endometriosis, defined as the presence of endometrial tissue outside the uterine cavity, is a benign chronic gynecological disorder. It is a common and estrogen-dependent gynecological disease that affects $10 \%$ of reproductive-aged women, mainly cause chronic pelvic pain and infertility.(1) There are many theories about the pathogenesis of endometriosis, such as coelomic epithelial metaplasia, immune defense deficiency and menstrual reflux. However, the exact physiopathology of endometriosis is unclear until now.(2)

Recent studies revealed that autophagy also plays an indispensable role in the physiological and pathophysiological processes related to endometriosis.(3) Autophagy is a process that includes subcellular membranes encapsulate cytoplasmic components, form autophagosomes and then fuse with lysosomes to form autolysosomes for degrading the contents.(4) It is a self-renewal pathway of cells that degrades damaged macromolecules and organelles to maintain cell metabolism and improve cell function. During autophagy, the metabolic wastes are selectively identified and isolated in double-membrane vesicles called autophagosomes, which are subsequently fused with acidic lysosomes containing hydrolases used for cargo degradation.(5) Autophagy could be induced by the nutrient depletion, oxidative stress, or other harmful conditions. Many pathological conditions such as cancer(6) and neurodegenerative diseases(7) are associated with the dysfunction of this process. Therefore, it is crucial for us to study the cellular autophagy-lysosomal on the pathogenesis as well as therapeutics of disorders.

TFEB, the member of the basic helix-loop-helix leucine-zipper family of transcription factors, has been identified as a master regulator of autophagic flux via inducing lysosome biogenesis and promoting autophagosome formation as well as its fusion with lysosome.(8-10) Several studies confirmed that the important role of TFEB in neurodegenerative(11) and renal cell carcinoma.(12) However, the role of TFEB in endometriosis hasn't be studied. This paper reviews the regulation mechanism of TFEB, and discusses its impact on the occurrence and development of endometriosis.

\section{Mechanisms regulating TFEB activity}

TFEB, together with MITF (melanogenesis associated transcription factor), TFE3 and TFEC constitute the MiT/TFE subfamily of transcription factors; it participates in cell metabolism and intracellular clearance by modulating the processes of lysosomal biogenesis, autophagy and lysosomal exocytosis. TFEB regulates biological processes by regulating the expression of downstream genes. The activity of TFEB is strictly controlled by post-translational modifications and protein-protein interactions.(13) Normally, TFEB is primarily located in cytoplasmic. Under the conditions of starvation, it is transported to the nucleus, binds to its target genes, promotes the transcription and expression of autophagy and lysosomal related proteins, which can improve the functions of autophagy and lysosome.(10) Recent studies have shown that TFEB and TFE3 not only respond to changes in nutritional levels, but also a variety of internal and external stress factors, including mitochondrial damage,(14) the accumulation of unfolded proteins in endoplasmic reticulum, (15) pathogens $(16,17)$ and physical exercise.(18) TFEB is mainly regulated post-translation via phosphorylation. Many factors involve in regulate TFEB through phosphorylation of specific amino acid residues. To date, at least six kinases, including mTOR, MAPK1,GSK3b, AKT, MAP4K3, and PKC $\beta$ have been identified to regulate TFEB function by phosphorylation. The activity of TFEB and TFE3 factors is negatively regulated by nutrient and growth factor-sensitive kinases (such as mTORC1, AKT, ERK2, and GSK3B) and positively regulated by the phosphatase calcineurin through the modulation of the phosphorylation status of multiple 
serine residues .(19, 20) Activated TFEB binds to promoter sequence named CLEAR sequenced elements to form CLEAR gene networks. This gene network enriches the genes encoding lysosomes endosomes and autophagy proteins. Therefore, stress-induced TFEB activation can adapt and expand the activity of the endosomal system by driving lysosomal biogenesis and autophagy flux.

The highly conserved sequence mTOR(mammalian target of rapamycin ) is an atypical serine/threonine kinase, belong to PI3K related protein kinases family.it is a key upstream kinase regulating autophagy(21) and also is a key upstream kinase that directly phosphorylates TFEB and inhibits its activity and expression.(22) It can phosphorylate two particular serine residues Ser142 and Ser211 in the TFEB protein, inducing the TFEB retained in cytoplasm, block the generation of new lysosomes, and decreases autophagic flux. Under conditions of amino acid satiety, Rag GTPases-Ragulator complex recruited TFEB to lysosomal membranes, and mammalian target of rapamycin complex 1 (mTORC1) phosphorylates TFEB at serine 211. the Phosphorylated TFEB is sequestered by chaperones of the 14-3-3 family, which actively prevent its translocation to the nucleus.(23) while Under starvation conditions, inactivation of mTORC1 allows nuclear translocation of TFEB to mediate cellular adaptation to stress. S142 is also dephosphorylated in the presence of MTORC1 inhibition,But its exact function is unclear. A recent study reported that S122 is the direct phosphorylation site of mTOR, which coordinates with S211 to regulate TFEB nuclear localization.(24) Therefore, mTOR can regulate TFEB by acting on different sites. Another serine/threonine phosphatase, calcineurin, is also involved in regulating TFEB activity, (25) In a state of nutritional deficiency or stress, Ca2+ is released from lysosomal MCOLN1 (a member of the transient receptor potential channel family), thereby exciting calcineurin, leading to TFEB dephosphorylation and nuclear translocation.(26) Serine/threonine kinase AMP-activated protein kinase (AMPK) complex is a sensor of energy in cells to regulate a variety of metabolic processes, including autophagy. It can regulate TFEB in different ways. AMPK can directly phosphorylate the upstream regulator TSC2 of mTOR and the mTORC1 subunit raptor. These two phosphorylation events lead to a decrease in mTOR activity,(27) which promoted TFEB nuclear translocation. This is a mTOR dependent way to activate TFEB. Recent studies have also shown that AMPK activates SIRT1(silent information regulator 1). SIRT1 is a highly conserved member of the histone deacetylase family, which can directly deacetylate TFEB (28) or indirectly activate TFEB via deacetylating the downstream protein PGC- $1 \alpha$ (peroxisome proliferator-activated receptor gamma, coactivator 1 alpha) .$(29,30)$ It is a coactivator that interacts with a broad range of transcription factors involved in various biological responses, including adaptive thermogenesis, mitochondrial biogenesis, oxidative metabolism and steroidogenesis. PGC-1a has a parallel effect on TFEB, overexpression of PGC-1a increased the abundance of TFEB protein, and knockout decreased the transcription and protein abundance of TFEB, $(31,32)$ such as In Huntington's disease, PGC- $1 \alpha$ promotes the elimination of protein aggregates by activating the transcription factor EB (TFEB).(33) These are mTOR independent pathways that activate TFEB. In addition, P53 has been shown to be positively correlated with TFEB, But the exact mechanism is unclear.

\section{TFEB and autophagy in endometriosis}

Endometriosis is characterized by the implantation of endometrial glands and stroma deep into the outside of the uterine cavity.(3) It mainly involves the ovaries and adjacent pelvic peritoneum. Endometriosis is not malignant, but its ability to infiltrate and invade distant tissues follows the same pattern as metastases from malignant tumors. As a chronic and estrogen-dependent disease, there are many theories about the pathogenesis of endometriosis, but no one can fully explain it. Autophagy has been a hot topic in recent years. It has also been reported to affect the production and invasion of ectopic endometrial tissues from endometriosis patients.(34) TFEB is a key factor in the formation of autophagic lysosomes. Roland P. Kuiper et al's study showed that TFEB was expressed in the endometrium, although its highest expression was in the placenta and lung.(35) Therefore, TFEB may play a role in the development of endometriosis. However, there are no reports about TFEB and endometriosis at home and abroad.

\section{1 ).autophagy in Endometriosis is down-regulated through the TFEB-mediated mTOR depen- dent pathway}

Many studies have shown that autophagy is down-regulated in endometriosis by detecting autophagy- 
related molecules and proteins. It is well known that inhibition of mTOR can activate autophagy, and the PI3K/AKT pathway is considered to be a positive regulator of mTOR activity.(36) Meanwhile, $\mathrm{PI} 3 \mathrm{~K} / \mathrm{AKT} / \mathrm{mTOR}$ pathway is a classic signaling pathway regulating autophagy. The PI3K/AKT/mTOR signaling pathway has been found to be closely related to endometriosis. Phosphorylation of various proteins in the PI3K/AKT/mTOR pathway leads to molecular interactions and ultimately the formation of endometriosis.(37) Guo J et al discovered that the phosphorylation level of mTOR in ectopic endometrium in patients with endometriosis was higher than that in eutopic endometrium.(38) Besides, inhibition of mTOR can alleviate the development of endometritis foci in rat/mouse models of endometriosis.(39) Moreover, in the mouse model established by Yan Liu et al, the inhibition of the PI3K/AKT/mTOR signaling pathway can alleviate endometriosis-associated sciatic nerve pain in a rat model of sciatic endometriosis, (40) Zhang L et al's study revealed that Beclin-1 mRNA and protein expression in eutopic and ectopic endometrium of patients with endometriosis was significantly reduced. Lei Zhan et al. studied autophagy marker proteins LC3 and P62 in the endometrium of endometriosis and leiomyoma. They found compared with the endometrium of leiomyoma group in the ectopic and eutopic endometrium of endometriosis groups, the expression of P62 was significantly higher. In comparison, the expressions of LC3 were down-regulated.(41) LC3 is proved as a credible marker of the autophagosome in mammalian cells. After the formation of autophagosome, LC3-I was subsequently combined with phospholipid-ethanolamine (PE) to form LC3-II, which was bound to the inner and outer membrane of autophagosome. Through binding directly to LC3, P62 /SQSTM1 selectively binds to autophagosomes and is effectively degraded by autophagy, therefore, the expression level of P62 was negatively correlated with autophagy activity.(42) All of these studies have revealed inadequate levels of autophagy in endometriosis. But the exact mechanism, however, is unclear. TFEB is a key factor in the formation of autophagic lysosomes. mTOR is the upstream signal molecule of TFEB, LC3II and p62 are the marker proteins after the autophagic lysosomes formation. Therefore, TFEB may play role in endometriosis tissue. The activated mTOR may phosphorylate the serine residues of TFEB and locate TFEB in the cytoplasm, thus preventing the formation of autophagic lysosome and cannot clearing the ectopic endometrium. In addition, Liang et al. found that there was a positive association between the expression of Beclin1 and TFEB, whereas TFEB silencing suppressed Beclin1 amplification. So the Low beclin-1 mRNA and protein expression in ectopic endometrium in patients with endometriosis may be caused by down-regulation of TFEB.(43)

\section{2).ROS leads to autophagy up-regulation of ovarian endometrioma through the TFEB- mediated mTOR independent pathway (figure).}

Ovarian endometriosis is widely accepted as the direct precursor of clear-cell and endometrioid ovarian carcinomas. $(44,45)$ However, when the researchers studied the level of autophagy in ovarian endometriosis, the results were different from endometriosis. Giulia Allavena et al. compared LC3 and P62 expression levels in normal endometrial tissues (NE), ovarian endometriotic cysts tissues(OMA) and eutopic endometrium (EEOMA) groups, they found that the LC3-II and LC3-II/LC3-I ratios in OMA were significantly higher than that in NE and EEOMA, while p62 was significantly lower in OMA than that in NE and EEOMA.(46) Ying Ding et al's study shows Beclin-1 expression of ovarian granule cells was increased and autophagy was enhanced in patients with ovarian endometrioma.(47) researchers also found that LC3B significantly increased expression in oviduct and ovarian endometrial epithelial lesions compared with secretory endometrial epithelium of the control group.(48) Serum CA125, which for the diagnosis and staging of ovarian endometriosis, was moderately positively correlated with LC3B protein expression.(49) All evidence is biased towards autophagy is up-regulation in ovarian endometriosis. However, this does not conflict with the down-regulation of autophagy observed in other areas of endometriosis. Because the increase of autophagy level may be considered as an integral part of the progression of endometriosis, which may contribute to the survival and damage maintenance of ectopic endometrial cells. Many studies indicate that oxidative stress positively associated with the proliferation and migration of endometrial cells in the peritoneal cavity, promoting endometriosis and infertility. Mitochondria is major source for ROS generation.(50) It is a class of molecules formed by incomplete reduction of oxygen, including superoxide anion $\left(\mathrm{O}_{2} *_{-}\right)$, hydrogen peroxide (H2O2), hydroxyl radical $\left({ }^{*} \mathrm{OH}\right)$, etc.(51) In endometriotic cysts, bleeding can occur inside the cyst during every men- 
struation cycle, so the cysts contain large amounts of free iron or non-protein-bound iron. Subsequently, the hemoglobin from an oxyhemoglobin state to the methemoglobin state through autoxidation and the Fenton reaction can produce excess ROS .(52) Oxidative stress caused by excess iron leads to uncontrolled cell growth and aberrant intracellular signaling.(53) It has been found that TFEB is activated under oxidative stress induced by tert-butyl peroxide or hydrogen peroxide in nematodes. $(54,55)$ On the contrary, ROS elimination inhibits TFEB activity and lysosomal function. (56-58) So the relation between ROS and TFEB may be a mechanism for ectopic endometriosis cell proliferation. ROS can regulate autophagy via TFEB in different ways. ROS overproduction can lead to the activation of autophagy by suppressing the PI3K/AKT/mTOR signaling pathway, which plays a critical role in regulating autophagy.(59) mTOR inactivation can dissociate TFEB from 14-3-3 and promote TFEB nuclear transposition to improve autophagy level. However ovarian endometriotic lesions have been shown to exhibit enhanced activation of mTOR compared with the normal endometrium.(60) José A et al reveal the ROS inducer sodium arsenite can activate TFEB and TFE3 without Inhibiting mTORC1 activity. This mechanism, they say, may enable some cell types that need both TFEB/TFE3 and mTORC1 signalings, such as immune cells or cancer cells, to survive and achieve robust cell growth in stressful conditions.(61) So there may be other mechanisms in ovarian endometriosis that do not depend on mTOR to control TFEB to upregulate autophagy. It was shown that under oxidative stress situations, AMPK is activated and regulated mitophagy to relieve intestinal ischemia/reperfusion-induced intestine disorder.(62) Abnormal AMPK expression was observed in the endometrial microenvironment,(63) phosphate-AMPK can activates SIRT1, SIRT1 deacetylation TFEB at K116, enhances the expression of autophagy/lysosomal related genes, and regulates the formation of autophagic vacuoles, $(28,64)$ study has found that SIRT1 was overexpressed in endometriosis patients and may be involved in the pathogenesis of endometriosis.(65) Moreover, Jung-yoon Yoo et al. found that in the baboon endometriosis model, SIRT1 protein was not significantly expressed in the endometrium of pre-inoculation (control) baboon. However, during the progression of endometriosis, SIRT1 protein levels increased significantly at 9 and 15 months after inoculation.(66) Therefore, the ROS-AMPK-SIRT1-TFEB pathway may be a mechanism for the progression of endometriosis. Besides, Izumi Suganuma et al. discovered PGC-1a was more highly expressed in ovarian endometrioma than in endometrium with endometriosis and normal endometrium, and PGC-1a can promote the proliferation of ovarian endometrioma stromal cells, whereas PGC-1a knockdown reduced the proliferation,(67) This may be due to SIRT1-mediated deacetylation of PGC-1a, which activates TFEB and thus induces autophagy and promotes ectopic endometrial cell proliferation. Besides, Recent studies suggest that the increasing level of ROS can directly activate lysosomal MCOLN1 to induce lysosomal Ca2+ release, establish a $\mathrm{Ca} 2+$ microdomain near the lysosome. This leads to calcineurin activation and TFEB dephosphorylation, which is no longer bind 14-3-3 proteins and can be freely translocated into the nucleus, where transcriptional activation of the lysosome/autophagy pathway occurs.$(26,68)$ what's more,Hongfeng Wang et al showed that ROS-dependent TFEB fast nuclear translocation could occur due to oxidation of TFEB on C212 without inhibiting the activity MTORC1.(69) Vitamin C is an antioxidant, with different doses of vitamin C (0.5 mg, $1.25 \mathrm{mg}$ and $2.5 \mathrm{mg}$ ), treatment of endometrial cyst model, Durak et al Found a significant reduction in weight and volume of cystic lesions in the $2.5 \mathrm{mg}$ vitamin $\mathrm{C}$ group at the end of treatment, suggesting that oxidative stress is involved in the progression of endometriosis(70). therefore, antioxidants may be a mechanism for the treatment of endometriosis, TFEB can be regulated by ROS through different mechanisms, so TFEB may also be a potential target for endometriosis treatment. What's more, compared with both NE and EEOMA, p53 is significantly down-regulated in OMA,(46) Study has shown that p53 deletion or the use of pifithrin- $\alpha$ chemical inhibition of p53 promotes the transfer of TFEB from cytoplasm to nucleus, thereby increasing the biogenesis of TFEB-mediated lysosomes and autophagosomes. Moreover, p53 re-expression down-regulates the TFEB target gene involved in autophagy-lysosome pathway, while TFEB silencing eliminates the regulatory effect of p53 on autophagy-lysosome pathway regulatory gene transcription.(71) So TFEB inhibitors may be more effective than antioxidants in treating endometriosis. Summary, there may be a variety of mechanisms in ovarian endometriosis cyst to regulate TFEB. TFEB activation and translocate into the nucleus acts on target genes, which can promote the expression of lysosome-related proteins, thus improving the level of autophagy of ectopic cells. High expression of TFEB and biogenesis of lysosome are markers of poor prognosis in a quarter of early breast cancer.(72) The expression of TFEB 
was positively correlated with the malignant progression of colorectal cancer. the tumor cells with high expression of TFEB had deeper invasion and higher lymph node metastasis rate.(43) Whether the level of TFEB associated with the severity of endometriosis? It requires further study.

\section{3). Difference from endometriosis and ovarian endometrioma}

Why do different researchers study the relationship between endometriosis and autophagy, but come to the opposite conclusion? We speculate that in the occurrence and development of endometriosis, there are mechanisms of oxidative stress and anti-oxidation. Reactive oxygen species (ROS) are produced continuously in normal cell metabolism, which are considered as signal transduction functions. However, when excessive ROS is induced by environmental factors or diseases, oxidative stress will occur, which leads to autophagy, apoptosis, necrosis,,and other physiological and pathological processes.(73) the accumulated ROS activate TFEB through mTOR independent mechanism and induce autophagy to maintain energy metabolism and remove excessive ROS. Because ovarian endometriosis can form cysts easier and lead to blood accumulation, which can produce more ROS. Ruiz, A et al. found that the expression of lc3b protein in endometriosis mouse model was decreased (compared with the uterine horn isolated from the same disease treatment), while the lc3b level in the epithelial cells and eutopic endometrium of patients with endometriosis was higher than that in the control group. They speculate that this difference may be due to the fact that the lesions in mice are equivalent to those in patients with early endometriosis. Because human diseases can't be diagnosed until a delay of 7-10 years.(48) This conjecture is similar to our hypothesis that autophagy is down-regulated in the early stage of endometriosis. As the disease progresses, the accumulation of ROS leads to the up regulation of autophagy, thus promoting the growth and infiltration of ectopic endometrial cells. Induction of autophagy may be beneficial to tumor prevention, Alternatively, it may promote tumor progression by enhancing autophagy-mediated intracellular circulation, which facilitates the removal of metabolic substrates and the preservation of mitochondrial functional pools.(8) There may be the same mechanism in occurrence and development of endometriosis that causes it to behave like a tumor.

\section{TFEB mediated autophagy upregulate to inhibit apoptosis in endometriosis}

Autophagy and apoptosis are the main factors determining cell fate. Apoptosis is a kind of cell death characterized by cell atrophy, chromatin condensation and other morphological changes. Autophagy, which means "self-feeding" is an important degradation pathway to eliminate protein aggregation and damaged organelles in cells.(74) More and more evidence show that apoptosis and autophagy have antagonistic effects. Autophagy helps human cancer cells survive through apoptotic resistance, and inhibition of autophagy leads to caspase-dependent apoptotic cell death.(75) In tumor microenvironment, inhibition of autophagy disrupts cell metabolism, causes genomic instability, interferes with differentiation, and destroys anti-cancer immune surveillance. During tumor development, cancer cells are exposed to different types of stress, including nutritional deficiency, metabolic stress and hypoxia, especially in the central region of the tumor. Autophagy can be reinstated which can increase the resistance of cancer cells to chemotherapy or radiotherapy.(76) The cytoprotective effect of autophagy can maintain the survival ability of tumor cells and prevent the apoptosis of cancer cells.(77) In nucleus pulposus cells, TFEB overexpression enhanced autophagy flux and lysosome function, which further protected nucleus pulposus cells from apoptosis and senescence induced by TBHP (ROS donor induced oxidative stress).(78) The same mechanism may exist in endometriosis. In early stage of endometriosis, inhibition of autophagy disrupts cell metabolism, interferes with differentiation, and evades immune surveillance. As the disease progresses, excessive ROS activates TFEB in different ways, and overexpression of TFEB promotes the expression of autophagy-related proteins, increases the autophagy flux and lysosome synthesis, thus maintaining cell growth and inhibiting apoptosis, leading to the worsening of endometriosis. It was reported that the expression of Bcl-2 was increased in ectopic endometriosis in ovarian endometriosis patients. Bcl-2 is an important anti-apoptotic regulator, and its increase is associated with impaired spontaneous apoptosis, indicating that apoptosis is decreased in endometrioma.(79)

\section{Conclusion and Perspectives}

In conclusion, autophagy plays an indispensable role in the occurrence and development of endometriosis. 
TFEB, as a key regulator of autophagy, its upstream and downstream signal molecules have abnormal expression in endometriosis. Moreover, apoptosis can be inhibited by TFEB mediated autophagy induction. Therefore, TFEB may be an important regulatory point of abnormal autophagy levels in endometriosis. At present, the treatment of endometriosis is still limited to hormone and surgery. However, hormone therapy is easy to relapse, and there are many side effects. Moreover, not all women with endometriosis have a response to hormonal therapy, and surgery is an invasive treatment. Therefore, it is necessary to find a new treatment for endometriosis, Autophagy is a good perspective for us to understand endometriosis, TFEB may also become a potential target for the treatment of endometriosis.

\section{Acknowledgment(s):}

We thank the staff of the Second Affiliated Hospital of Wenzhou Medical University,We are also highly thankful to the previous research for providing valuable ideas for this review.

Disclosure: The authors declare that no competing interests exist.

Contribution to authorship: Zhang Q and Zhao Y identified this theme. Yao Chen, Chenxi Dai and Zongwen Liang earched for literature. Chen QY and Zhu SN wrote the manuscript draft. Zhang Q and Zhao Y revised the manuscript. All authors read and approved the final manuscript

Funding source: This review was financially supported by the Natural Science Foundation of Zhejiang Province [grant No. LY20H040005], the Medical and Health Research Project of Zhejiang Province of China [grant No. 2017KY479、2018KY127、2018KY520].

\section{Reference:}

1. Gadducci A, Zannoni GF. Endometriosis-associated Extraovarian Malignancies: A Challenging Question for the Clinician and the Pathologist. Anticancer Res. 2020;40(5):2429-38. Epub 2020/05/06. doi: 10.21873/anticanres.14212. PubMed PMID: 32366386.

2. Lagana AS, Garzon S, Gotte M, Vigano P, Franchi M, Ghezzi F, et al. The Pathogenesis of Endometriosis: Molecular and Cell Biology Insights. Int J Mol Sci. 2019;20(22). Epub 2019/11/14. doi: 10.3390/ijms20225615. PubMed PMID: 31717614; PubMed Central PMCID: PMCPMC6888544.

3. Zhan L, Li J, Wei B. Autophagy in endometriosis: Friend or foe? Biochem Biophys Res Commun. 2018;495(1):60-3. Epub 2017/11/07. doi: 10.1016/j.bbrc.2017.10.145. PubMed PMID: 29107692.

4. Hansen M, Rubinsztein DC, Walker DW. Autophagy as a promoter of longevity: insights from model organisms. Nat Rev Mol Cell Biol. 2018;19(9):579-93. Epub 2018/07/15. doi: 10.1038/s41580-018-0033-y. PubMed PMID: 30006559; PubMed Central PMCID: PMCPMC6424591.

5. Kuo C-J, Hansen M, Troemel E. Autophagy and innate immunity: Insights from invertebrate model organisms. Autophagy. 2018;14(2):233-42. doi: 10.1080/15548627.2017.1389824.

6. White E. The role for autophagy in cancer. Journal of Clinical Investigation. 2015;125(1):42-6. doi: $10.1172 /$ jci73941.

7. Stamatakou E, Wróbel L, Hill SM, Puri C, Son SM, Fujimaki M, et al. Mendelian neurodegenerative disease genes involved in autophagy. Cell Discovery. 2020;6(1). doi: 10.1038/s41421-020-0158-y.

8. Bahrami A, Bianconi V, Pirro M, Orafai HM, Sahebkar A. The role of TFEB in tumor cell autophagy: Diagnostic and therapeutic opportunities. Life Sci. 2020;244:117341. Epub 2020/01/24. doi: 10.1016/j.lfs.2020.117341. PubMed PMID: 31972208.

9. Pastore N, Brady OA, Diab HI, Martina JA, Sun L, Huynh T, et al. TFEB and TFE3 cooperate in the regulation of the innate immune response in activated macrophages. Autophagy. 2016;12(8):1240-58. Epub 2016/05/14. doi: 10.1080/15548627.2016.1179405. PubMed PMID: 27171064; PubMed Central PMCID: PMCPMC4968228. 
10. Raben N, Puertollano R. TFEB and TFE3: Linking Lysosomes to Cellular Adaptation to Stress. Annu Rev Cell Dev Biol. 2016;32:255-78. Epub 2016/06/15. doi: 10.1146/annurev-cellbio-111315-125407. PubMed PMID: 27298091; PubMed Central PMCID: PMCPMC6490169.

11. Cortes CJ, La Spada AR. TFEB dysregulation as a driver of autophagy dysfunction in neurodegenerative disease: Molecular mechanisms, cellular processes, and emerging therapeutic opportunities. Neurobiol Dis. 2019;122:83-93. Epub 2018/06/01. doi: 10.1016/j.nbd.2018.05.012. PubMed PMID: 29852219; PubMed Central PMCID: PMCPMC6291370.

12. Calio A, Brunelli M, Segala D, Pedron S, Remo A, Ammendola S, et al. Comprehensive analysis of 34 MiT family translocation renal cell carcinomas and review of the literature: investigating prognostic markers and therapy targets. Pathology. 2020;52(3):297-309. Epub 2020/02/29. doi: 10.1016/j.pathol.2019.11.006. PubMed PMID: 32107074.

13. Napolitano G, Ballabio A. TFEB at a glance. J Cell Sci. 2016;129(13):2475-81. Epub 2016/06/03. doi: 10.1242/jcs.146365. PubMed PMID: 27252382; PubMed Central PMCID: PMCPMC4958300.

14. Nezich CL, Wang C, Fogel AI, Youle RJ. MiT/TFE transcription factors are activated during mitophagy downstream of Parkin and Atg5. J Cell Biol. 2015;210(3):435-50. Epub 2015/08/05. doi: 10.1083/jcb.201501002. PubMed PMID: 26240184; PubMed Central PMCID: PMCPMC4523611.

15. Martina JA, Diab HI, Brady OA, Puertollano R. TFEB and TFE3 are novel components of the integrated stress response. EMBO J. 2016;35(5):479-95. Epub 2016/01/28. doi: 10.15252/embj.201593428. PubMed PMID: 26813791; PubMed Central PMCID: PMCPMC4772850.

16. Gray MA, Choy CH, Dayam RM, Ospina-Escobar E, Somerville A, Xiao X, et al. Phagocytosis Enhances Lysosomal and Bactericidal Properties by Activating the Transcription Factor TFEB. Curr Biol. 2016;26(15):1955-64. Epub 2016/07/12. doi: 10.1016/j.cub.2016.05.070. PubMed PMID: 27397893; PubMed Central PMCID: PMCPMC5453720.

17. Ouimet M, Koster S, Sakowski E, Ramkhelawon B, van Solingen C, Oldebeken S, et al. Mycobacterium tuberculosis induces the miR-33 locus to reprogram autophagy and host lipid metabolism. Nat Immunol. 2016;17(6):677-86. Epub 2016/04/19. doi: 10.1038/ni.3434. PubMed PMID: 27089382; PubMed Central PMCID: PMCPMC4873392.

18. Pastore N, Vainshtein A, Klisch TJ, Armani A, Huynh T, Herz NJ, et al. TFE3 regulates whole-body energy metabolism in cooperation with TFEB. EMBO Mol Med. 2017;9(5):605-21. Epub 2017/03/12. doi: 10.15252/emmm.201607204. PubMed PMID: 28283651; PubMed Central PMCID: PMCPMC5412821.

19. Puertollano R, Ferguson SM, Brugarolas J, Ballabio A. The complex relationship between TFEB transcription factor phosphorylation and subcellular localization. EMBO J. 2018;37(11). Epub 2018/05/17. doi: 10.15252/embj.201798804. PubMed PMID: 29764979; PubMed Central PMCID: PMCPMC5983138.

20. Hsu CL, Lee EX, Gordon KL, Paz EA, Shen WC, Ohnishi K, et al. MAP4K3 mediates amino aciddependent regulation of autophagy via phosphorylation of TFEB. Nat Commun. 2018;9(1):942. Epub 2018/03/07. doi: 10.1038/s41467-018-03340-7. PubMed PMID: 29507340; PubMed Central PMCID: PMCPMC5838220.

21. Rabanal-Ruiz Y, Otten EG, Korolchuk VI. mTORC1 as the main gateway to autophagy. Essays Biochem. 2017;61(6):565-84. Epub 2017/12/14. doi: 10.1042/EBC20170027. PubMed PMID: 29233869; PubMed Central PMCID: PMCPMC5869864.

22. Ugolino J, Ji YJ, Conchina K, Chu J, Nirujogi RS, Pandey A, et al. Loss of C9orf72 Enhances Autophagic Activity via Deregulated mTOR and TFEB Signaling. PLoS Genet. 2016;12(11):e1006443. Epub 2016/11/23. doi: 10.1371/journal.pgen.1006443. PubMed PMID: 27875531; PubMed Central PMCID: PMCPMC5119725.

23. Zhitomirsky B, Yunaev A, Kreiserman R, Kaplan A, Stark M, Assaraf YG. Lysosomotropic drugs activate TFEB via lysosomal membrane fluidization and consequent inhibition of mTORC1 activity. Cell Death Dis. 
2018;9(12):1191. Epub 2018/12/14. doi: 10.1038/s41419-018-1227-0. PubMed PMID: 30546014; PubMed Central PMCID: PMCPMC6294013.

24. Silvia Vega-Rubin-de-Celis1, Samuel Pena-Llopis1, 2, Meghan Konda1, 2, James Brugarolas1, 2*. Multistep regulation of TFEB by MTORC1. Mar 4 2017. doi: 10.1080/15548627.2016.1271514.

25. Tong Y, Song F. Intracellular calcium signaling regulates autophagy via calcineurin-mediated TFEB dephosphorylation. Autophagy. 2015;11(7):1192-5. Epub 2015/06/06. doi: 10.1080/15548627.2015.1054594. PubMed PMID: 26043755; PubMed Central PMCID: PMCPMC4590610.

26. Medina DL, Di Paola S, Peluso I, Armani A, De Stefani D, Venditti R, et al. Lysosomal calcium signalling regulates autophagy through calcineurin and TFEB. Nat Cell Biol. 2015;17(3):288-99. Epub 2015/02/28. doi: 10.1038/ncb3114. PubMed PMID: 25720963; PubMed Central PMCID: PMCPMC4801004.

27. Herzig S, Shaw RJ. AMPK: guardian of metabolism and mitochondrial homeostasis. Nat Rev Mol Cell Biol. 2018;19(2):121-35. Epub 2017/10/05. doi: 10.1038/nrm.2017.95. PubMed PMID: 28974774; PubMed Central PMCID: PMCPMC5780224.

28. Huang J, Wang X, Zhu Y, Li Z, Zhu YT, Wu JC, et al. Exercise activates lysosomal function in the brain through AMPK-SIRT1-TFEB pathway. CNS Neurosci Ther. 2019;25(6):796-807. Epub 2019/03/14. doi: 10.1111/cns.13114. PubMed PMID: 30864262; PubMed Central PMCID: PMCPMC6515701.

29. Liang D, Zhuo Y, Guo Z, He L, Wang X, He Y, et al. SIRT1/PGC-1 pathway activation triggers autophagy/mitophagy and attenuates oxidative damage in intestinal epithelial cells. Biochimie. 2020;170:1020. doi: 10.1016/j.biochi.2019.12.001.

30. Tang S, Fang Y, Huang G, Xu X, Padilla-Banks E, Fan W, et al. Methionine metabolism is essential for SIRT

1-regulated mouse embryonic stem cell maintenance and embryonic development. The EMBO Journal. 2017;36(21):3175-93. doi: 10.15252/embj.201796708.

31. Erlich AT, Brownlee DM, Beyfuss K, Hood DA. Exercise induces TFEB expression and activity in skeletal muscle in a PGC-1 $\alpha$-dependent manner. American Journal of Physiology-Cell Physiology. 2018;314(1):C62C72. doi: 10.1152/ajpcell.00162.2017.

32. Mansueto G, Armani A, Viscomi C, D'Orsi L, De Cegli R, Polishchuk EV, et al. Transcription Factor EB Controls Metabolic Flexibility during Exercise. Cell Metabolism. 2017;25(1):182-96. doi: 10.1016/j.cmet.2016.11.003.

33. Tsunemi T, Ashe TD, Morrison BE, Soriano KR, Au J, Roque RAV, et al. PGC-1 Rescues Huntington's Disease Proteotoxicity by Preventing Oxidative Stress and Promoting TFEB Function. Science Translational Medicine. 2012;4(142):142ra97-ra97. doi: 10.1126/scitranslmed.3003799.

34. Sachiko Matsuzaki 1 J-LP, 2 Michel Canis 1, 2. In vitro and in vivo effects of MK2206 and chloroquine combination therapy on endometriosis: Autophagy may be required for regrowth of endometriosis. 2018 May. doi: 10.1111/bph.14170.

35. Kuiper RP, Schepens M, Thijssen J, Schoenmakers EF, van Kessel AG. Regulation of the MiTF/TFE bHLH-LZ transcription factors through restricted spatial expression and alternative splicing of functional domains. Nucleic Acids Res. 2004;32(8):2315-22. Epub 2004/05/01. doi: 10.1093/nar/gkh571. PubMed PMID: 15118077; PubMed Central PMCID: PMCPMC419459.

36. Shi B, Ma M, Zheng Y, Pan Y, Lin X. mTOR and Beclin1: Two key autophagy-related molecules and their roles in myocardial ischemia/reperfusion injury. Journal of Cellular Physiology. 2019;234(8):12562-8. doi: $10.1002 /$ jcp. 28125 . 
37. Zhou R, Cao Y, Ye Q, Zhuang M, Xie S, Zhong R, et al. Ginsenoside Rg3 inhibits angiogenesis in a rat model of endometriosis through the VEGFR-2-mediated PI3K/Akt/mTOR signaling pathway. Plos One. 2017;12(11). doi: 10.1371/journal.pone.0186520.

38. Guo J, Gao J, Yu X, Luo H, Xiong X, Huang O. Expression of DJ-1 and mTOR in Eutopic and Ectopic Endometria of Patients with Endometriosis and Adenomyosis. Gynecologic and Obstetric Investigation. 2015;79(3):195-200. doi: 10.1159/000365569.

39. Kacan T, Yildiz C, Baloglu Kacan S, Seker M, Ozer H, Cetin A. Everolimus as an mTOR Inhibitor Suppresses Endometriotic Implants: an Experimental Rat Study. Geburtshilfe Frauenheilkd. 2017;77(1):6672. Epub 2017/02/14. doi: 10.1055/s-0042-115566. PubMed PMID: 28190891; PubMed Central PMCID: PMCPMC5283166.

40. Liu Y, Qin X, Lu X, Jiang J. Effects of inhibiting the PI3K/Akt/mTOR signaling pathway on the pain of sciatic endometriosis in a rat model. Canadian Journal of Physiology and Pharmacology. 2019;97(10):963-70. doi: 10.1139/cjpp-2019-0156.

41. Zhan L, Yao S, Sun S, Su Q, Li J, Wei B. NLRC5 and autophagy combined as possible predictors in patients with endometriosis. Fertility and Sterility. 2018;110(5):949-56. doi: 10.1016/j.fertnstert.2018.06.028.

42. Jiang P, Mizushima N. LC3- and p62-based biochemical methods for the analysis of autophagy progression in mammalian cells. Methods. 2015;75:13-8. Epub 2014/12/09. doi: 10.1016/j.ymeth.2014.11.021. PubMed PMID: 25484342.

43. Liang J, Jia X, Wang K, Zhao N. High expression of TFEB is associated with aggressive clinical features in colorectal cancer. Onco Targets Ther. 2018;11:8089-98. Epub 2018/12/07. doi: 10.2147/OTT.S180112. PubMed PMID: 30519051; PubMed Central PMCID: PMCPMC6239122.

44. Anglesio MS, Bashashati A, Wang YK, Senz J, Ha G, Yang W, et al. Multifocal endometriotic lesions associated with cancer are clonal and carry a high mutation burden. J Pathol. 2015;236(2):201-9. Epub 2015/02/19. doi: 10.1002/path.4516. PubMed PMID: 25692284; PubMed Central PMCID: PMCPMC6680210.

45. Kurman RJ, Shih Ie M. The Dualistic Model of Ovarian Carcinogenesis: Revisited, Revised, and Expanded. Am J Pathol. 2016;186(4):733-47. Epub 2016/03/26. doi: 10.1016/j.ajpath.2015.11.011. PubMed PMID: 27012190; PubMed Central PMCID: PMCPMC5808151.

46. Allavena G, Carrarelli P, Del Bello B, Luisi S, Petraglia F, Maellaro E. Autophagy is upregulated in ovarian endometriosis: a possible interplay with p53 and heme oxygenase-1. Fertil Steril. 2015;103(5):124451 e1. Epub 2015/03/17. doi: 10.1016/j.fertnstert.2015.02.007. PubMed PMID: 25772769.

47. Ding Y, Zhu Q, He Y, Lu Y, Wang Y, Qi J, et al. Induction of autophagy by Beclin-1 in granulosa cells contributes to follicular progesterone elevation in ovarian endometriosis. Translational Research. 2020. doi: 10.1016/j.trsl.2020.06.013.

48. Ruiz A, Rockfield S, Taran N, Haller E, Engelman RW, Flores I, et al. Effect of hydroxychloroquine and characterization of autophagy in a mouse model of endometriosis. Cell Death Dis. 2016;7:e2059. Epub 2016/01/19. doi: 10.1038/cddis.2015.361. PubMed PMID: 26775710; PubMed Central PMCID: PMCPMC4816166 submitted" (MN, IF). All other co-authors declare no conflict of interest.

49. Jingjie Zheng M, PhD1,*, Xishao Luo, MD2,*, Jiaping Bao, MD2, Xiaowang Huang, MD2, Yi Jin, MD3, Lin Chen, BS3, and Feiyun Zheng, BS2. Decreased Expression of HOXA10 May Activate the Autophagic Process in Ovarian Endometriosis. 2018. doi: 10.1177/1933719118768704.

50. Chen C, Zhou Y, Hu C, Wang Y, Yan Z, Li Z, et al. Mitochondria and oxidative stress in ovarian endometriosis. Free Radical Biology and Medicine. 2019;136:22-34. doi: 10.1016/j.freeradbiomed.2019.03.027. 
51. Prasad S, Gupta SC, Tyagi AK. Reactive oxygen species (ROS) and cancer: Role of antioxidative nutraceuticals. Cancer Letters. 2017;387:95-105. doi: 10.1016/j.canlet.2016.03.042.

52. Iwabuchi T, Yoshimoto C, Shigetomi H, Kobayashi H. Oxidative Stress and Antioxidant Defense in Endometriosis and Its Malignant Transformation. Oxid Med Cell Longev. 2015;2015:848595. Epub 2015/07/18. doi: 10.1155/2015/848595. PubMed PMID: 26185594; PubMed Central PMCID: PMCPMC4491397.

53. Mori M, Ito F, Shi L, Wang Y, Ishida C, Hattori Y, et al. Ovarian endometriosis-associated stromal cells reveal persistently high affinity for iron. Redox Biol. 2015;6:578-86. Epub 2015/10/27. doi: 10.1016/j.redox.2015.10.001. PubMed PMID: 26498255; PubMed Central PMCID: PMCPMC4633839.

54. Lin XX, Sen I, Janssens GE, Zhou X, Fonslow BR, Edgar D, et al. DAF-16/FOXO and HLH-30/TFEB function as combinatorial transcription factors to promote stress resistance and longevity. Nat Commun. 2018;9(1):4400. Epub 2018/10/26. doi: 10.1038/s41467-018-06624-0. PubMed PMID: 30353013; PubMed Central PMCID: PMCPMC6199276.

55. Ma S, Fang Z, Luo W, Yang Y, Wang C, Zhang Q, et al. The C-ETS2-TFEB Axis Promotes Neuron Survival under Oxidative Stress by Regulating Lysosome Activity. Oxid Med Cell Longev. 2016;2016:4693703. Epub 2016/05/20. doi: 10.1155/2016/4693703. PubMed PMID: 27195074; PubMed Central PMCID: PMCPMC4853961.

56. Wu B, Tan M, Cai W, Wang B, He P, Zhang X. Arsenic trioxide induces autophagic cell death in osteosarcoma cells via the ROS-TFEB signaling pathway. Biochem Biophys Res Commun. 2018;496(1):16775. Epub 2018/01/09. doi: 10.1016/j.bbrc.2018.01.018. PubMed PMID: 29307831.

57. Xuesong Li1 XZ, 3, Longbin Zheng1, Jiayuan Kou1, Zhaoyu Zhong1, Yueqing Jiang1, Wei Wang3, Zengxiang Dong3, Zhongni Liu1, Xiaobo Han1, Jing Li4, Ye Tian*,1,5, Yajun Zhao*,1 and Liming Yang*. Hypericin-mediated sonodynamic therapy induces autophagy and decreases lipids in THP-1 macrophage by promoting ROS-dependent nuclear translocation of TFEB. 2016. doi: 10.1038/cddis.2016.433.

58. Zeng W, Xiao T, Cai A, Cai W, Liu H, Liu J, et al. Inhibiting ROS-TFEB-Dependent Autophagy Enhances Salidroside-Induced Apoptosis in Human Chondrosarcoma Cells. Cellular Physiology and Biochemistry. 2017;43(4):1487-502. doi: 10.1159/000481971.

59. Portal-Núñez S, Esbrit P, Alcaraz MJ, Largo R. Oxidative stress, autophagy, epigenetic changes and regulation by miRNAs as potential therapeutic targets in osteoarthritis. Biochemical Pharmacology. 2016;108:110. doi: 10.1016/j.bcp.2015.12.012.

60. Choi J, Jo M, Lee E, Kim HJ, Choi D. Differential induction of autophagy by mTOR is associated with abnormal apoptosis in ovarian endometriotic cysts. Molecular Human Reproduction. 2013;20(4):309-17. doi: 10.1093/molehr/gat091.

61. Martina JA, Puertollano R. Protein phosphatase 2A stimulates activation of TFEB and TFE3 transcription factors in response to oxidative stress. Journal of Biological Chemistry. 2018;293(32):12525-34. doi: 10.1074/jbc.RA118.003471.

62. Jin Wena BX, Yuchao Suna, Mengqiao Liana, Yanli Lia, Yuan Lina, ${ }^{*}$, Dapeng Chenb,Yunpeng Diaoa,*, Marwan Almoiliqya, Li Wang. Paeoniflorin protects against intestinal ischemia/reperfusion by activating LKB1/AMPK and promoting autophagy. 2019 Aug. doi: 10.1016/j.phrs.2019.104308.

63. Machado DE, Perini JA, de Mendonca EM, Branco JR, Rodrigues-Baptista KC, Alessandra-Perini J, et al. Clotrimazole is effective for the regression of endometriotic implants in a Wistar rat experimental model of endometriosis. Molecular and Cellular Endocrinology. 2018;476:17-26. doi: 10.1016/j.mce.2018.04.005.

64. Bao J, Zheng L, Zhang Q, Li X, Zhang X, Li Z, et al. Deacetylation of TFEB promotes fibrillar $\mathrm{A} \beta$ degradation by upregulating lysosomal biogenesis in microglia. Protein \& Cell. 2016;7(6):417-33. doi: 10.1007/s13238-016-0269-2. 
65. Teasley HE, Beesley A, Kim TH, Risinger J, Young SL, Jeong J-W, et al. Differential Expression of KRAS and SIRT1 in Ovarian Cancers with and Without Endometriosis. Reproductive Sciences. 2020;27(1):145-51. doi: 10.1007/s43032-019-00017-4.

66. Jung-Yoon Yoo1, Tae Hoon Kim1, Asgerally T. Fazleabas1,3, Wilder A. Palomino4, Soo Hyun Ahn5, Chandrakant Tayade5, David P. Schammel6, Steven L. Young7, Jae-Wook Jeong1,3 \& Bruce A. Lessey8. KRAS Activation and overexpression of SIRT1/BCL6 Contributes to the Pathogenesis of Endometriosis and Progesterone Resistance. 2017 Jul. doi: 10.1038/s41598-017-04577-w.

67. Hisashi Kataoka TM, Hiroyuki Okimura, Hiroshi Matsushima, Fumitake Ito, Akemi Koshiba, Yukiko Tanaka, Kanoko Akiyama, Eiko Maeda, Takuya Sugahara, Yosuke Tarumi, Izumi Kusuki, Khaleque N Khan, and Jo Kitawaki. Peroxisome proliferator-activated receptor- $\gamma$ coactivator $1 \alpha$-mediated pathway as a possible therapeutic target in endometriosis. 2019 Jun 4. doi: 10.1093/humrep/dez067.

68. Zhang X, Cheng X, Yu L, Yang J, Calvo R, Patnaik S, et al. MCOLN1 is a ROS sensor in lysosomes that regulates autophagy. Nature Communications. 2016;7(1). doi: 10.1038/ncomms12109.

69. Wang H, Wang N, Xu D, Ma Q, Chen Y, Xu S, et al. Oxidation of multiple MiT/TFE transcription factors links oxidative stress to transcriptional control of autophagy and lysosome biogenesis. Autophagy. 2019;16(9):1683-96. doi: 10.1080/15548627.2019.1704104.

70. Durak Y, Kokcu A, Kefeli M, Bildircin D, Celik H, Alper T. Effect of vitamin C on the growth of experimentally induced endometriotic cysts. J Obstet Gynaecol Res. 2013;39(7):1253-8. Epub 2013/05/31. doi: 10.1111/jog.12050. PubMed PMID: 23718930.

71. Zhang Z, Wang H, Ding Q, Xing Y, Xu D, Xu Z, et al. The tumor suppressor p53 regulates autophagosomal and lysosomal biogenesis in lung cancer cells by targeting transcription factor EB. Biomedicine \& Pharmacotherapy. 2017;89:1055-60. doi: 10.1016/j.biopha.2017.02.103.

72. Giatromanolaki A, Sivridis E, Kalamida D, Koukourakis MI. Transcription Factor EB Expression in Early Breast Cancer Relates to Lysosomal/Autophagosomal Markers and Prognosis. Clinical Breast Cancer. 2017;17(3):e119-e25. doi: 10.1016/j.clbc.2016.11.006.

73. He L, He T, Farrar S, Ji L, Liu T, Ma X. Antioxidants Maintain Cellular Redox Homeostasis by Elimination of Reactive Oxygen Species. Cell Physiol Biochem. 2017;44(2):532-53. Epub 2017/11/18. doi: 10.1159/000485089. PubMed PMID: 29145191.

74. Liu J, Liu W, Yang H. Balancing Apoptosis and Autophagy for Parkinson's Disease Therapy: Targeting BCL-2. ACS Chemical Neuroscience. 2018;10(2):792-802. doi: 10.1021/acschemneuro.8b00356.

75. Chen Y, Henson ES, Xiao W, Huang D, McMillan-Ward EM, Israels SJ, et al. Tyrosine kinase receptor EGFR regulates the switch in cancer cells between cell survival and cell death induced by autophagy in hypoxia. Autophagy. 2016;12(6):1029-46. doi: 10.1080/15548627.2016.1164357.

76. Marino G, Niso-Santano M, Baehrecke EH, Kroemer G. Self-consumption: the interplay of autophagy and apoptosis. Nat Rev Mol Cell Biol. 2014;15(2):81-94. Epub 2014/01/10. doi: 10.1038/nrm3735. PubMed PMID: 24401948; PubMed Central PMCID: PMCPMC3970201.

77. Mukhopadhyay S, Panda PK, Sinha N, Das DN, Bhutia SK. Autophagy and apoptosis: where do they meet? Apoptosis. 2014;19(4):555-66. doi: 10.1007/s10495-014-0967-2.

78. Zheng G, Pan Z, Zhan Y, Tang Q, Zheng F, Zhou Y, et al. TFEB protects nucleus pulposus cells against apoptosis and senescence via restoring autophagic flux. Osteoarthritis and Cartilage. 2019;27(2):347-57. doi: 10.1016/j.joca.2018.10.011.

79. Wang C, Jin A, Huang W, Tsang LL, Cai Z, Zhou X, et al. Up-regulation of Bcl-2 by CD147 Through ERK Activation Results in Abnormal Cell Survival in Human Endometriosis. The Journal of Clinical Endocrinology \& Metabolism. 2015;100(7):E955-E63. doi: 10.1210/jc.2015-1431. 


\section{Legends for figures:}

Title :TFEB-mediated autophagy in ovarian endometriosis

AMPK:Serine/threonine kinase AMP-activated protein kinase

SIRT1: silent information regulator 1

PGC-1a:peroxisome proliferator-activated receptor gamma, coactivator 1 alpha

Description: In ovarian endometriosis, free iron stimulates ROS production and activates TFEB in ways that independent of mTOR. Firstly, ROS can directly oxidize TFEB. Secondly, ROS can promote the release of lysosomal Ca2+ through MCOLN1, Ca2+ activate calcineurin which can dephosphorylate TFEB. In addition, ROS can regulate sirt-1 through AMPK, which can activate TFEB directly or through PGC-1. The activated or dephosphorylated TFEB can enters the nucleu and promote the transcription and expression of lysosome and autophagy related genes, form autophagic lysosome, phagocytize metabolic wastes and abnormal organelles, release amino acids and recycled by cells, which can lead to cell proliferation and endometriosis infiltration.

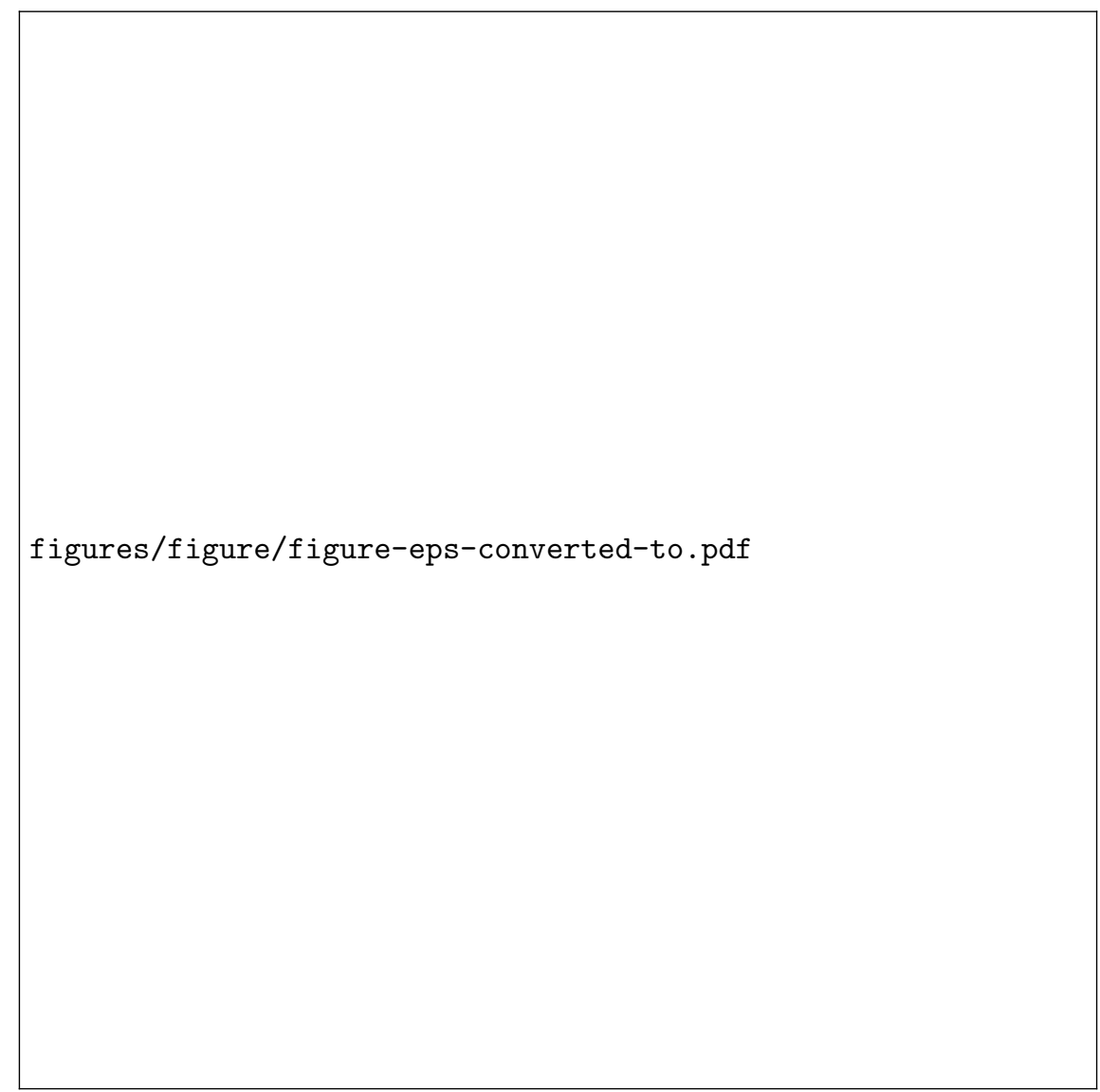

